\title{
A Rainfall Distribution for the Lampao Site in the Chi River Basin, Thailand
}

\author{
Bungon Kumphon ${ }^{1 *}$, Arun Kaew-Man ${ }^{1}$, Parakorn Seenoi ${ }^{2}$ \\ ${ }^{1}$ Department of Mathematics, Mahasarakham University, Mahasarakham, Thailand \\ ${ }^{2}$ Department of Statistics, Khon Kaen University, Khon Kaen, Thailand \\ Email: *bungon.k@msu.ac.th
}

Received August 12, 2012; revised September 10, 2012; accepted October 11, 2012

\begin{abstract}
In this study, the four-parameter kappa distribution with L-Moments estimation has been used to fit the distribution of weekly rainfall data at Lampao in the Chi River Basin, Thailand. The weekly precipitations with probabilities 0.75 were estimated, and the extreme rainfall estimates obtained can be used for water and agriculture management.
\end{abstract}

Keywords: L-Moments; Four Parameters Kappa Distribution; Goodness of Fit Test

\section{Introduction}

This paper deals with precipitation frequency analysis in the central area of Northeast Thailand. Under the Koppen classification the Northeast climate is called tropical wet-dry or tropical savannah. The two main mechanisms that support the rainfall in this region are the southwest monsoon during May to October, and the tropical cyclone depression July to September. Both are more important for agriculture and in the design and construction of various flood control measures, including the operational management of reservoirs. Reliable estimates of rainfall quantities at specified recurrence intervals by operational hydro-meteo-rologists have therefore been one issue. Ref [1] investigated the distribution of monthly rainfall in Northeast Thailand, and found the data could be fitted by either a Gamma distribution or a log-normal distribution. A more recent study by [2] showed that the incomplete Gamma distribution fits the daily rainfall in Thailand, where the rainfall starts from week 12 up to between week 20 and week 29, and then increases up to between week 36 and week 39, with very heavy rainfall in the Northeast, East and Southern regions.

In general, maximum likelihood estimation (MLE) is too sensitive to investigate extreme values. From our experience with a quasi-Newton algorithm, we find that a term in the logarithm function goes negative at some points $x_{i}$, and the MLE is computationally inefficient. The L-Moments approach developed by [3] has been widely used for parameter estimation because it provides robust and reliable parameter estimation, particularly

*Corresponding author. from small samples. It is also computationally more tractable than MLE, and less sensitive to outliers that may be present in the sample due to severe drought, flood or cyclone events. Further, L-Moments are linear combinations of ranked observations and do not involve squares or cubes, as in the more conventional methods for moment estimators. The four-parameter kappa distribution (K4D) also introduced by [4] is a very general distribution form that includes a variety of distributions-e.g. the generalized extreme value distribution, the generalized Pareto distribution, the generalized logistic distribution, the Gumbel distribution, etc. Several applications and examples see [5-7]. Ref [8] also used the K4D with LMoments estimation (L-ME), in modelling Indian monsoon rainfall. Ref [9] found that L-ME and MLE worked equally well for the three parameter kappa distribution.

The K4D with L-Moments procedure is adopted in this paper, to fit the distribution for the rainfall in a part of the Chi River Basin-more precisely, on the weekly precipitation data between 1984 to 2010 for the upper North Eastern region, obtained at the Lampao telemetering information station and the remote stations of the Hydrology and Water Management Center, Royal Irrigation department (HWMC) located around the Lampao reservoir area. The set of 0.75 probability estimates of the rainfall then obtained for each week are applicable to crop water management.

\section{Study Area and Data}

The Lampao River is a branch of the Chi River, and extends for $262 \mathrm{~km}$ but carries rather little water-viz. ap- 
proximately $9300 \mathrm{~km}^{3}$ of water per annum. One headwayter in the Nongharn District (Udonthanee Province) runs south through five districts in Kalasin Province, to meet the Chi River in the Kammalasai District, Kalasin Province-cf. Figure 1. In wet seasons, flash floods in the floodplain of the Chi River basin can be a concern. However, despite many local drought areas and the low quality of the soil, these areas produce sticky rice and other cash crops such as manioc (Cassava) and sugar cane, and the main income for the population is from agriculture. Consequently, rainfall and water management are of key importance for agriculturists in these areas given the low rainfall and potential water shortages during the growing season. On the other hand, there can be flooding due to heavy rains towards the end of the rainy season. The Lampao Reservior, built during 1963 to 1968 and storing $1430 \mathrm{mcm}^{3}$ of water, assists in agriculture over 314,300 rais $(50,288$ hectares) in the harvest season and 180,000 rais (28,800 hectares) in the dry season, and in flood prevention. More than 30,000 households around this river and the reservoir system stand to gain from effective water management.

The daily rainfall data from the nine telemetering information stations located around the reservoir (one master station; TP1, and eight remote stations; from TP2 to TP10, where TP7 is nearby TP1, so we decided to study the master station) was employed in the analysis. The data were processed according to the water year, beginning on 1st April and ending on 31st March the following year, as recorded over the 26 years between 1984 and 2010. The definition of light rain is water in drops of between 0.1 to $10 \mathrm{~mm}$ in diameter [10]. The amount of rainfall at a station was then obtained by summing up the

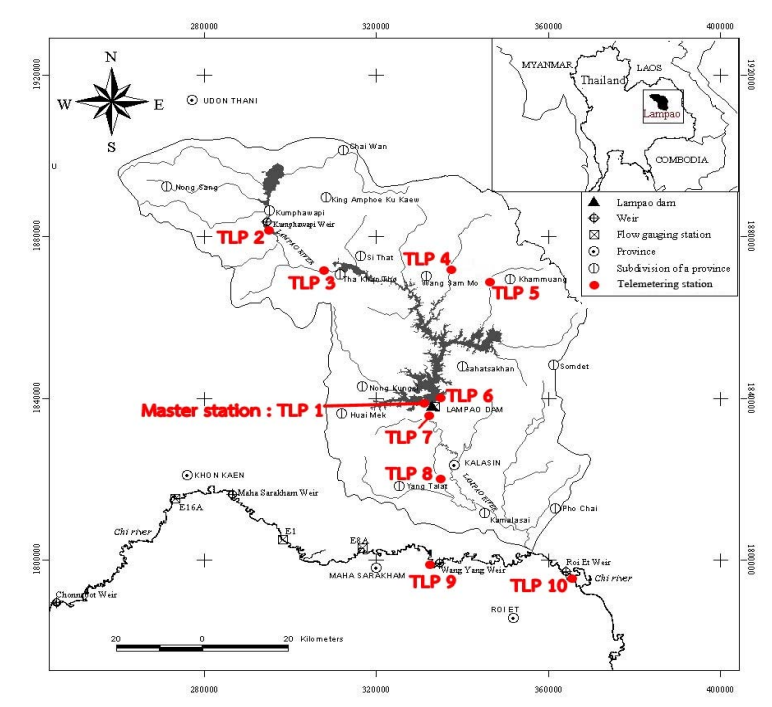

Figure 1. Map of Lampao reservoir and the measuring of telemetering stations in the Lampao River. daily amounts $(>0.1 \mathrm{~mm})$ in that week for the fitted distribution. For the rainfall at a probability equal to 0.75 , which is the optimal value for effective rainfall and irrigation planning $[11,12]$, were also calculated for TLP3 station because this is a first station at the head of reservoir, cover the largest area of the irrigation.

\section{Backgrond Theory}

L-Moments are summary statistics for probability distributions and data samples, analogous to ordinary moments [3]. They provide measures of location, dispersion, skewness, kurtosis, and other aspects of the shape of probability distributions or data samples. Using the uniform distribution function as its foundation and based on shifted Legendre polynomials, each statistical L-Moment is computed linearly (hence the $\mathrm{L}$ reference), giving a more robust estimate for a given amount of data than other methods. The sampling properties for L-Moments statistics are nearly unbiased, even in small samples, and are near normally distributed. These properties make them well suited for characterizing environmental data that commonly exhibit moderate to high skewness.

For the random variables of sample size $n$ drawn from the distribution of a random variable $X$ with the mean $m$ and variance $s^{2}$, the cumulative distribution function of the $\mathrm{K} 4 \mathrm{D}$ for $k \neq 0, h \neq 0$ is

$$
F(x)=\left\{1-h[1-k(x-\xi) / \alpha]^{1 / k}\right\}^{1 / h}
$$

The probability density function is

$$
f(x)=\alpha^{-1}[1-k(x-\xi) / \alpha]^{(1 / k)-1} \times[F(x)]^{1-h}
$$

for $-\infty<x<\infty$ and $\alpha>0$ where $\xi$ is a location parameter, $\alpha$ is a scale parameter and $h, k$ are the shape parameters (skewness and kurtosis), respectively. The quantile function (inverse cumulative distribution function) is

$$
x(F)=\xi+\frac{\alpha}{k}\left[1-\left(\frac{1-F^{h}}{h}\right)^{k}\right]
$$

Special cases of Equation (1) take the form of different distribution functions, such as the generalized Pareto distribution, the generalized extreme value distribution or the generalized logistic distribution when $k \neq 0$ and $h=$ $1,0,-1$ respectively. In the same way, for $k=0$ and $h=1$, $0,-1$, we have the exponential distribution, the Gumbel distribution and the logistic distribution, respectively; and for $k=1$ and $h=1,0$, the distributions are the uniform distribution and reverse exponential distribution, respectively.

Let $X_{1: 1}, \cdots, X_{1: n}$, be the order statistics such that the L-Moments of $X$ are defined by 


$$
\lambda_{r}=r^{-1} \sum_{k=0}^{r-1}(-1)^{k}\left(\begin{array}{c}
r-1 \\
k
\end{array}\right) E\left(X_{r-k: r}\right), r=1,2, \cdots
$$

where $r$ is the $r$ th L-Moment of a distribution and $E\left(X_{i: r}\right)$ is the expected value of the $i$ th smallest observation in a sample of size $r$. The first four L-Moments of a random variable $X$ can be written as

$$
\begin{aligned}
& \lambda_{1}=E(X), \\
& \lambda_{2}=\frac{1}{2} E\left(X_{2: 2}-X_{1: 2}\right), \\
& \lambda_{3}=\frac{1}{3} E\left(X_{3: 3}-2 X_{2: 3}+X_{1: 3}\right), \\
& \lambda_{4}=\frac{1}{4} E\left(X_{4: 4}-3 X_{3: 4}+3 X_{2: 4}-X_{1: 4}\right) .
\end{aligned}
$$

[3] demonstrated the utility of estimators based on the L-Moment ratios in hydrological extreme analysis. The second moment is often scaled by the mean, so that a coefficient of variability is determined-viz,

$$
\tau=L-C_{v}=\frac{\lambda_{2}}{\lambda_{1}}
$$

where $\lambda_{1}$ is the measure of location. As with the definitions and the meaning of the ratios between ordinary moments, the coefficients of L-kurtosis and L-skewness are defined as $\tau_{r}=\frac{\lambda_{r}}{\lambda_{2}}$ for $r \geq 3$, where $\tau_{3}$ is the measure of skewness (L-Cs) and $\tau_{4}$ is the measure of kurtosis (L-Ck).

\section{Application to Weekly Rainfall}

The exact distribution of parameter estimators obtained by this method is difficult to derive in general, so we explored the fit between the theoretical distribution (K4D) and the real data set, as shown in Figure 2. Parameters and a goodness of fit test via the Kolmogorov-Smirnovtest (KS) and the Anderson-Darling test (AD) with a 0.05 significance level computed for the data at all nine stations are tabulated in Table $\mathbf{1}$ and shown that K4D fit well with all the rainfall for nine stations. A close inspection of the parameters shows values of the respective parameters $h>0$ and $k \neq 0$, suggesting that the underlying distribution tends towards the generalized Pareto distribution rather than the generalized extreme value distribution for all stations.

As an application of this methodology to the estimation of the maximum amount of rainfall at the 0.75 probability each week, the example observations were shown at one station above the reservoir within the Chi Basin using the parameters in Equations (1) and (2) estimated at TLP3 station. The estimated values have been computed as presented in Table 2. The highlighted area

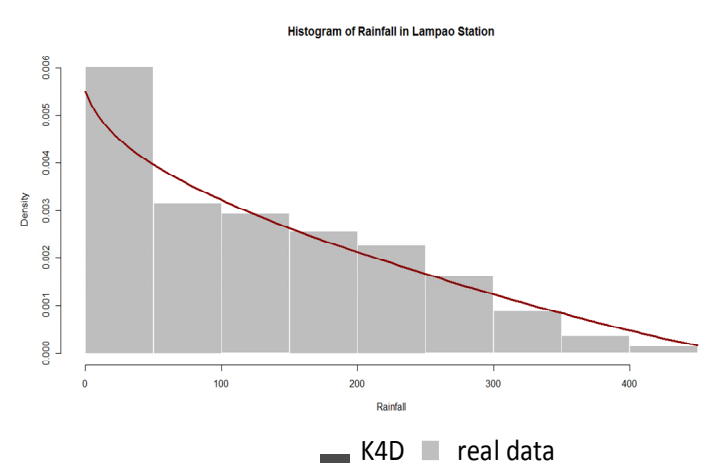

Figure 2. The exploration for goodness of fit for K4D at TLP3 station.

Table 1. Parameters estimation and goodness of fit test for the K4D distribution.

\begin{tabular}{ccccccc}
\hline site & location & scale & $h$ & $k$ & KS (p-value) & AD (p-value) \\
\hline TLP1 & -23.355 & 169.427 & 0.273 & 1.165 & $0.280(0.953)$ & $0.0402(0.887)$ \\
TLP2 & -14.444 & 176.833 & 0.321 & 1.088 & $0.240(0.975)$ & $0.0360(0.943)$ \\
TLP3 & -37.121 & 237.376 & 0.455 & 1.127 & $0.061(0.267)$ & $1.370(0.211)$ \\
TLP4 & -18.790 & 178.192 & 0.292 & 1.101 & $0.320(0.923)$ & $0.031(0.988)$ \\
TLP5 & -18.872 & 166.970 & 0.378 & 1.121 & $0.243(0.974)$ & $0.031(0.990)$ \\
TLP6 & 26.010 & 146.238 & 0.283 & 0.787 & $0.535(0.711)$ & $0.037(0.933)$ \\
TLP8 & 26.051 & 112.908 & 0.157 & $0.7310 .3880(0.861)$ & $0.040(0.943)$ \\
TLP9 & 20.012 & 152.246 & 0.294 & 0.819 & $0.717(0.544)$ & $0.043(0.812)$ \\
TLP 10 & 48.534 & 117.765 & 0.136 & 0.538 & $1.101(0.308)$ & $0.051(0.649)$ \\
\hline
\end{tabular}

Table 2. The estimation of the maximum amount of rainfall for each week at the 0.75 probability, TLP3 station.

\begin{tabular}{cccccc}
\hline Week & Amount & Week & Amount & Week & Amount \\
\hline 1 & 0.000 & 19 & 61.487 & 37 & 73.535 \\
2 & 0.000 & 20 & 61.835 & 38 & 62.461 \\
3 & 0.000 & 21 & 76.256 & 39 & 37.234 \\
4 & 0.000 & 22 & 69.715 & 40 & 49.580 \\
5 & 0.305 & 23 & 65.838 & 41 & 39.601 \\
6 & 0.000 & 24 & 72.143 & 42 & 22.817 \\
7 & 1.566 & 25 & 74.050 & 43 & 4.943 \\
8 & 0.000 & 26 & 60.660 & 44 & 4.855 \\
9 & 5.483 & 27 & 64.666 & 45 & 0.000 \\
10 & 0.000 & 28 & 56.508 & 46 & 0.039 \\
11 & 1.659 & 29 & 65.155 & 47 & 0.000 \\
12 & 16.165 & 30 & 41.593 & 48 & 0.000 \\
13 & 23.785 & 31 & 79.242 & 49 & 0.000 \\
14 & 10.443 & 32 & 82.184 & 50 & 0.000 \\
15 & 16.638 & 33 & 103.833 & 51 & 0.000 \\
16 & 19.828 & 34 & 103.358 & 52 & 0.000 \\
17 & 43.136 & 35 & 84.370 & & \\
18 & 45.875 & 36 & 110.496 & & \\
\hline
\end{tabular}

shows the rainy season from week 19 to week 40. The 
awareness weeks are weeks 33 to 36 , with very heavy rain (84 to $110 \mathrm{~mm}$.). This is useful information for the hydro-meteorologists, and for reservoir planners who manage water release and storage before and during the awareness weeks 33 to 36 . The best weeks for agriculturists to plan drying processes for crops are 1 to 6,8 , 10 and 45 to 52 because there is then no rainfall.

\section{Conclusion}

For the planning and design of crop scheduling and the design of water management in Northeast Thailand, the distribution of weekly rainfall was investigated from data on weekly rainfall for a part of Chi River Basin surrounding the Lampao Reservoir. The data is fitted well by K4D with L-Moments estimation, and there is some evidence for a generalized Pareto distribution. There is usually one distribution that passes the goodness-of-fit test. Although, there might be more than one distribution for this relatively small region (from the family of such distributions). For the estimated rainfall at the specific probability 0.75 , there is low to no rainfall in the dry season, which is the best time for drying crops or any associated activity that has no water requirement. On the other hand, there is very high value of rainfall in the rainy season.

\section{Acknowledgements}

Financial support was provided by the Faculty of Science, and Mahasarakham University Development Fund, Mahasarakham University. The data was provided by the Hydrology and Water Management Center for the upper north eastern Royal Irrigation department, Thailand. The author thanks Professor Roger Hosking for his support and helpful suggestions.

\section{REFERENCES}

[1] H. N. Phien, A. Arbhabhirama and A. Sunchindah, "Distribution of Monthly Rainfall in Northeast Thailand," Southeast Asian Studies, Vol. 18, No. 1, 1980, pp. 110-
122.

[2] A. Phayomjamsri, "Weekly Rainfall Probability for Thailand,” Study and Research Division, Meteorological Dapartment, Bangkok, 2008.

[3] J. R. M. Hosking, "L-Moments: Analysis and Estimation of Distribution Using Linear Combination of Order Statistics," Journal of the Royal Statistical Society Series B, Vol. 52, No. 1, 1990, pp. 105-124.

[4] J. R. M. Hosking, “The Four-Parameter Kappa Distribution," IBM Journal of Research Development, Vol. 38, No. 3, 1994, pp. 251-258. doi:10.1147/rd.383.0251

[5] E. S. S. Eslamian and H. Feizih, "Maximum Monthly Rainfall Analysis Using L-Moments for an Arid Region in Isfahan Province, Iran," Journal of Applied Meteorology and Climatology, Vol. 46, 2007, pp. 494-503. doi:10.1175/JAM2465.1

[6] J. S. Park and H. S. Jung, "Modelling Korean Extreme Rainfall Using a Kappa Distribution,” Biometrika, Vol. 79, 2002, pp. 827-832.

[7] J. R. M. Hosking and J. R. Wallis, "Regional Frequency Analysis. An Approach Based on L-Moments," Cambridge University Press, Cambridge, New York, Melbourne, 1997. doi:10.1017/CBO9780511529443

[8] B. P. Parida, "Modelling of Indian Summer Monsoon Rainfall Using a Four-Parameter Kappa Distribution,” International Journal of Climatology, Vol. 19, No. 12, 1999, pp. 1389-1398. doi:10.1002/(SICI)1097-0088(199910)19:12<1389::AIDJOC435>3.0.CO;2-T

[9] J. S. Park, S. C. Seo and T. Y. Kim, “A Kappa Distribution with a Hydrological Application," Stochastic Environmental Research and Risk Assessment, Vol. 23, No. 5, 2009, pp. 579-586. doi:10.1007/s00477-008-0243-5

[10] Thai Meteorological Department, “Thai Weather,” 2007. http://www.tmd.go.th/info/info.php?FileID=22

[11] N. G. Dastane, "Effective Rainfall in Irrigated Agriculture,” FAO Irrigation, and Drainage Paper No. 25, Rome, 1977.

[12] L. R. Oldeman and M. Frere, "A Study of the Agroclimatology of the Humid Tropics of Southeast Asia,” Technical Note No. 179, Food and Agriculture Organisation of the United Nations, 1982, pp. 72-76. 